\title{
Variação dos Elementos Keplerianos Angulares de Satélites Artificiais Lunares Devido à Ação de Forças que Derivam de um Potencial Gravitacional
}

\author{
Rodolpho Vilhena de Moraes \\ Maria Lívia Galhego T. X. da Costa* \\ Instituto de Ciência e Tecnologia, Universidade Federal de São Paulo \\ 12231-289, São José dos Campos, SP \\ E-mails: rodolpho.vilhena@gmail.com, livia.thibes@gmail.com, \\ Antônio Fernando Bertachini de Almeida Prado \\ Divisão de Mêcanica Espacial e Controle, Instituto Nacional de Pesquisas Espaciais \\ 12227-010, São José dos Campos, SP \\ E-mail: prado@dem.inpe.br.
}

\section{RESUMO}

Tendo em vista diversos interesses, entre eles, científicos, econômicos ou políticos, missões espaciais têm sido enviadas ao espaço para explorar corpos do Sistema Solar e, em especial, à Lua, desde a corrida espacial, na segunda metade do século passado. Atualmente, existe um número crescente de missões planejadas para explorar corpos extraterrestres, incluindo as que envolvem veículos espaciais orbitando satélites naturais de outros planetas [1]. Para o sucesso de tais, é necessário, porém, conhecer o comportamento orbital de satélites artificiais, quando perturbados por forças conservativas e não-conservativas. Neste trabalho, são analisadas as variações dos elementos keplerianos de órbitas de satélites artificiais orbitando luas naturais, considerando, somente, os efeitos provocados pela distribuição não-uniforme de massa do corpo central. Os corpos celestes considerados são: Lua, Europa, Ganimedes e Titã. Ênfase é dada ao comportamento das variáveis angulares.

Quando se estuda o movimento de satélites artificiais ao redor de luas de planetas, levando-se em conta a distribuição não-homogênea de massa do corpo central e os polinômios de Legendre para descrever o potencial gravitacional, observa-se que nem sempre a ordem de grandeza de alguns coeficientes associados à ordem e grau dos polinômios é hierarquicamente proporcional à ordem e grau dos polinômios.

Por exemplo, diferentemente do caso da Terra, para a Lua (satélite natural da Terra), a ordem do coeficiente associado ao $C_{22}$ é apenas um décimo menor que o coeficiente associado ao $J_{2}$. Também, podemos citar a ordem de grandeza do coeficiente associado ao $J_{9}$ é maior que a ordem de grandeza do coeficiente associado ao $J_{3}$ [2]. Isto faz com que o movimento orbital de satélites artificiais ao redor da Lua, sob alguns aspectos, seja diferente do movimento orbital de satélites artificiais terrestres [3].

O objetivo deste trabalho é comparar as variações dos elementos orbitais de satélites artificiais orbitando a Lua, Europa, Ganimedes e Titã, devido à distribuição não-uniforme de massa dos mesmos, com as variações dos elementos orbitais de satélites artificiais terrestres devido à distribuição não-uniforme de massa do corpo central.

A metodologia utilizada é a seguinte: a) considerando, para cada caso, o plano de referência sendo o do equador do corpo central, o potencial gravitacional é desenvolvido em termos dos polinômios de Legendre e expresso em função dos elementos orbitais [4];

* bolsista de Iniciação Científica PIBIC/CNPq 
b) o potencial é substituído nas equações planetárias de Lagrange e uma solução analítica aproximada é obtida, fornecendo expressões analíticas para variações temporais dos elementos orbitais do satélite artificial; c) simulando algumas condições iniciais para satélites artificiais orbitando satélites planetários (Lua, Europa, Ganimedes e Titã), valores numéricos para tais variações são obtidos; d) os resultados obtidos são analisados e comparados.

Palavras-chave: Satélites Planetários, Potencial Gravitacional, Elementos Orbitais, Satélites Artificiais.

\section{Referências}

[1] NASA Missions/NASA, https ://www.nasa.gov/missions

[2] J. Y. Chen, J. S. Ning, C. Y. Zhang, J. Lou, On the determination of lunar gravity field in the Chinese first lunar prospector mission. Chinese Journal of Geophysics, vol. 48, ${ }^{\circ}$ 2, pp. 275-281, 2005.

[3] J. P. de S. Carvalho, R. Vilhena de Moraes, A. F. B. A. Prado, Planetary Satellite Orbiters: Applications for the Moon. Mathematical Problems in Engineering doi:10.1155/2011/187478, 2011.

[4] B. Morando, "Mouvement d'un satellite artificiel de la Terre", Gordon \& Breach, Paris, 1974. 